\title{
Stevens, Tim, und Nicholas Michelsen (Hrsg.) (2020): Pessimism in International Relations: Provocations, Possibilities, Politics
}

\author{
London: Palgrave Macmillan. 226 Seiten. $£ 54,99$
}

\author{
Sebastian Schindler $\mathbb{D}$
}

Angenommen: 23. September 2021 / Online publiziert: 12. Oktober 2021

(C) Der/die Autor(en) 2021

Pessimismus beschreibt eine Haltung zur Welt, die das Schlimmste annimmt. Die zwei Londoner Politikwissenschaftler Tim Stevens und Nicholas Michelsen haben eine höchst nützliche Essay-Sammlung herausgegeben, die die Bedeutung dieser Haltung in der Gegenwart und Vergangenheit, im politischen Denken und in der Politik erörtert. Pessimismus - das ist ihr Ausgangspunkt - ist heute weitverbreitet. Aber es ist alles andere als klar, wie dieser Pessimismus normativ und politisch einzuordnen ist. Man hört pessimistische Äußerungen aus dem Munde Donald Trumps (,The world is a total mess.“) genauso wie von jungen Menschen, die freitags für eine klimagerechte Zukunft protestieren. Mithilfe des von Stevens und Michelsen herausgegebenen Sammelbandes kann man auf Spurensuche gehen und die Formenvielfalt pessimistischer Haltungen ergründen.

Am eindrücklichsten weist Elisabetta Brighi in ihrem Beitrag auf die Ambivalenz von Pessimismus hin. Brighi beginnt ihren Aufsatz mit dem Verweis auf die Bedeutung pessimistischer Ansichten im gegenwärtigen Rechtspopulismus, der Angst und Furcht kultiviert und daraus Aggressionen und Angriffe rechtfertigt. Später schildert sie, dass negatives Denken für Kritische Theoretiker der Frankfurter Schule wie etwa Herbert Marcuse eine zentrale Rolle spielt (S. 105). Immer wieder schwankt Brighi hin und her zwischen dem reaktionären und emanzipativen Charakter der negativen Weltsicht. Sie zieht eine Verbindung zwischen Pessimismus (d.h. der Erwartung von Verlust und Niederlage) und Ressentiment (welches Verlust und Niederlage auf eine unfaire Behandlung durch andere zurückführt, S. 103). Selbst das Ressentiment kann emanzipatorisch wirken; es ist, wie auch die Emotion des Ärgers, eine mögliche Quelle von Kritik, die zu mehr Gerechtigkeit führen kann - Robert Solomon spricht gar von einer ,passion for justice denied“ (zit. auf S. 107), die im

Sebastian Schindler $(\bowtie)$

Ludwig-Maximilians-Universität München, München, Deutschland

E-Mail: sebastian.schindler@gsi.uni-muenchen.de 
Herzen der Demokratie stünde. Dann wiederum diskutiert Brighi ausführlich Siegmund Freud und sie zitiert Wendy Browns Kritik an der gegenwärtigen Linken, die sich laut Brown mehr für ihre eigene Unmöglichkeit interessiere als für ihre potenzielle Fruchtbarkeit (S. 112). Negative Gefühle über den Zustand der Welt sind ambivalent und es ist Brighis Verdienst, das in der gegenwärtigen politischen Lage und ideenhistorisch versiert herausgearbeitet zu haben. Jedoch bleibt man als Leser oder Leserin recht allein mit der wichtigen Frage zurück, wie und ob es möglich ist, zwischen mehr oder weniger emanzipatorischen Arten des negativen Fühlens zu unterscheiden.

Anders als Brighi schlagen sich die meisten anderen Beiträge in Stevens' und Michelsens Buch auf die eine oder andere Seite und diskutieren entweder mehr emanzipatorische oder mehr reaktionäre Äußerungen pessimistischer Weltsichten. Kurioserweise arbeitet Stevens einerseits selbst gemeinsam mit Michelsen die reaktionäre Variante des Pessimismus in einem Kapitel zum ,alt right knowledge“Diskurs heraus. Das ist allerdings nicht durchweg überzeugend ausgearbeitet, etwa wenn die beiden Herausgeber behaupten, dass der Kampf der Neuen Rechten gegen die dominante Kultur ,immer“ mit einem Aufruf zum Pessimismus gegenüber dem Gesagten, Gehörten, Erwarteten und allen Erklärungen beginne (S. 123). Ist hier wirklich noch spezifisch von Pessimismus die Rede? Wo sind die begrifflichen Grenzen zu anderen Phänomenen wie Skeptizismus oder Post-truth? Andererseits arbeitet Stevens auf durchaus feine und überzeugende Weise die produktive Variante des Pessimismus in einem Kapitel zur „survival research“ des klassischen Realisten John Herz heraus. Herz, der in den Internationalen Beziehungen bis heute vor allem durch seinen klassischen Aufsatz zum Sicherheitsdilemma bekannt ist, arbeitete in seinen letzten Lebensjahrzehnten auf eine Transformation derjenigen Kategorien hin, die heute klassischerweise dem sogenannten realistischen Denken zugeschrieben werden. Mit seiner „Überlebens-Forschung“ ging es ihm nicht primär um das individuelle Überleben von Staaten im internationalen System, sondern um die tiefere Erkenntnis, dass ökologische und nukleare Bedrohungen das Überleben der gesamten Menschheit infrage stellen. Nicht Reifizierung von Interessen, sondern die verschütt und versteckt liegende Einsicht, dass unser aller Überlebensinteresse gleichermaßen und durch die gleichen Kräfte bedroht ist - das wollte Herz zeigen und damit eine Veränderung von Wissenschaft und Gesellschaft gleichermaßen anregen. Was Stevens hier zeigt, ist, dass Pessimismus wachrütteln und zu transformativen Handlungen anstiften kann: ,it is precisely because this form of pessimism envisions dark futures that it demands action in the present to avoid them" (S. 84). Herz selbst floh aufgrund seines realistischen Pessimismus, der die Machtergreifung der Nazis vorausahnte, rechtzeitig aus Deutschland und überlebte. Vielleicht, so schließt Stevens' überzeugender Essay, kann Pessimismus heute, im Zeitalter des menschengemachten Klimawandels, auch unser Leben retten (S. 94).

Sein Pessimismus motivierte Herz, rechtzeitig die Flucht vor den Nazis zu ergreifen; zugleich führte ihr Pessimismus die Nazis in die Katastrophe, wenn man Mark Baileys Aufsatz folgen will, der sich mit dem Mythos-Begriff Ernst Cassirers auseinandersetzt. In Baileys Aufsatz begegnen wir wiederum der reaktionären Variante des Pessimismus, die aus der Wahrnehmung gegenwärtiger Gefahren nicht die Notwendigkeit zu integrativer Kooperation und Überlebensforschung, sondern im Gegenteil 
einen Aufruf zu Gewalt und Vernichtung ableitet, der letztlich im Tod aller Politik als Auseinandersetzung mit anderen endet (S. 57). Cassirer war vom Mythos-Begriff so fasziniert, weil er ihm geeignet schien, um den überraschend schnellen Erfolg der Nazis und das Ende der Weimarer Republik zu erklären (S. 54). Bailey leitet aus Cassirers Analysen nicht zuletzt eine Warnung vor gegenwärtigen Formen des Pessimismus ab, die den Glauben an die moderne Rationalität zu schnell und in zu verallgemeinerter Form aufgegeben haben - etwa dem Poststrukturalismus (S. 62). Dessen Pessimismus ähnelt laut Bailey der Lebensphilosophie (die er amüsanterweise durchweg als „Liebensphilosophie“ im englischen Text zitiert), die die moderne Rationalität ganz allgemein verantwortlich macht für das Übel in der Welt, nicht zuletzt wegen ihrer lebensfremden Trennung von Geist und Körper.

Bei allen kleinen und größeren Mängeln liefert „Pessimism in International Relations: Provocations, Possibilities, Politics “ insgesamt eine durchaus wertvolle Sammlung von Aufsätzen, die die Auseinandersetzung mit einer heute weitverbreiteten Haltung zur Welt inspirieren kann. Ich habe hier nur einige Kapitel ausführlicher besprechen können, aber schon diese Auswahl macht deutlich, dass Pessimismus keineswegs die Abwesenheit von Hoffnung auf Veränderung beinhalten muss. Stattdessen kann Pessimismus auch einfach nur das Bewusstsein beinhalten, dass kein Wandel endgültig ist und grundlegende Probleme gewöhnlich nicht für immer verschwinden, sondern sich in jeder Zeit und für jede Generation neu stellen (siehe dazu auch den Beitrag Richard Ned Lebow, S. 15). Die Hoffnung auf eine qualitative Veränderung fehlt allenfalls bei den extrem reaktionären Äußerungen von Pessimismus, die man vielleicht eher als Fatalismus oder Zynismus bezeichnen könnte. Im Fall Trump etwa ist ziemlich klar, dass er aus der zynischen Behauptung, Washington sei ein korrupter Sumpf, zugleich die Erlaubnis ableitet, selbst zynisch und korrupt zu handeln, weil die Welt eben so sei und sich nie ändern werde. Genau an diesem Punkt, denke ich, könnte eine Unterscheidung zwischen dem reaktionär-zynischen und dem produktiv-emanzipatorischen Pessimismus sinnvoll festgemacht werden. Optimismus ohne Pessimismus mag naiv sein; Pessimismus ohne Optimismus ist zynisch.

Funding Open Access funding enabled and organized by Projekt DEAL.

Open Access Dieser Artikel wird unter der Creative Commons Namensnennung 4.0 International Lizenz veröffentlicht, welche die Nutzung, Vervielfältigung, Bearbeitung, Verbreitung und Wiedergabe in jeglichem Medium und Format erlaubt, sofern Sie den/die ursprünglichen Autor(en) und die Quelle ordnungsgemäß nennen, einen Link zur Creative Commons Lizenz beifügen und angeben, ob Änderungen vorgenommen wurden.

Die in diesem Artikel enthaltenen Bilder und sonstiges Drittmaterial unterliegen ebenfalls der genannten Creative Commons Lizenz, sofern sich aus der Abbildungslegende nichts anderes ergibt. Sofern das betreffende Material nicht unter der genannten Creative Commons Lizenz steht und die betreffende Handlung nicht nach gesetzlichen Vorschriften erlaubt ist, ist für die oben aufgeführten Weiterverwendungen des Materials die Einwilligung des jeweiligen Rechteinhabers einzuholen.

Weitere Details zur Lizenz entnehmen Sie bitte der Lizenzinformation auf http://creativecommons.org/ licenses/by/4.0/deed.de. 